\title{
Evaluation of the constructive state of conservation of historical structures. A new method of operating analysis: the ANVIV protocol.
}

\author{
Domenico Colapietro*, Fabio Fatiguso**, Marco Pinto*** \\ *DICATECh, Politecnico di Bari, Bari, Italy \\ **DICATECh, Politecnico di Bari, Bari, Italy \\ ***Studio COLAPIETRO: Ingegneria Strutturale \& Consulting, Bari, Italy
}

\section{ABSTRACT}

The conservation status assessment of historical traditional architecture on extended range increasingly requires analysis tools, which allow to synthesize and process parameters of interest for the formulation of an evaluation on their state of conservation. An appropriate prevention strategy development of, based on the loss risk assessment, seems the most appropriate way to address the management problem of similar structures. This approach requires simplified analysis tools, which allow to synthesize and process parameters of interest for the formulation of a current state evaluation of these structures, that can ensure, in a coordinated manner, an immed iate conservation status assessment. This paper shows the first results of use of a protocol named ANVIV which allows acquisition and interpolation of constructive and decay information for masonry buildings with diffe rent geometric configuration in plan and elevation. It has been developed as part of a research project, and is able to quickly show the physical conservation state of these buildings, both locally and globally, highlighting critical issues and planning appropriate intervention programs.
\end{abstract}

Keywords: Build ing Information, Conservation Status Assessment, Deterioration, Historical Bu ild ings, Local and Global Analysis, Vu lnerability.

\section{INTRODUCTION}

An increasingly percentage of building works in Italy concerns existing artifacts, this is because both building areas are limited and Italian buildings are often characterized by good architectural and constructive quality that makes it profitable to recover them, in order to extend their life and adapt them to the needs of modern living. Besides these motivations it is also spreading a greater awareness of the historical and architectural importance of the old town centers, the beauty of their landscapes and the importance of their development, which results in a greater sensitivity to the conservation topic. The interesting and fascinating aspect of the old villages, usually consisting of formally very simple architectures, a church, and sometimes a mansion, is in the richness and complexity of public and collective spaces that have come to form in the centuries from overlapping of countless building interventions. A spontaneous texture, therefore, grew from a medieval layout that has changed the landscape using the same materials and colors, a long time consolidated insertion that made them a harmonious whole.

Recovery is return in possession of what has been lost, the reacquisition of a disappeared condition, and it is usually a complex action that must be able to combine respect of the existing (materials, forms, meanings, history) with the needs of current users, taking into account the available resources and capabilities, seeking a balance between the different instances with the help of different disciplines.

The development of prevention strategies both seismic and of global asset maintenance for traditional architectures seems the most appropriate way to deal with the management problem of them. In that direction, a particularly important point is the risk of loss evaluation, which is considered as a reference for any necessary operational tool aimed at identifying priorities and allocation of resources [1].

The risk assessment increasingly requires analysis tools which enable to synthesize and process the parameters of interest for the formulation of a conservation status assessment of the building, by limiting the detail analysis, through the aid of mathematical numerical models, to the next step, only for those structures for which a further deployment of resources is really needed [2]. All this creates the basis for the development of new pre-analysis instruments that can ensure, in a coordinated manner, an immediate state of risk assessment for traditional architectures, from the individual to the group of buildings, through which plan really necessary recovery interventions. 


\section{METHODOLOGY}

The present paper proposes the development of a protocol, called ANVIV (Analysis and Evaluation of Intrinsic Vu Inerability) [3] for the acquisition and interpolation of constructive and decay information for each sub-system connoting a masonry building with structure of different geometrical configuration in plan and in elevation. This protocol allows to quickly evaluate the state of physical preservation of these buildings, both locally if analyzed in its individual parts, and globally if compared with other build ings.

The ANVIV protocol starts from the awareness that the state of local or global physical conservation of historical masonry buildings, can be evaluated by the disarticulation of them in three main technological sub-systems: vertical and horizontal bearing structures, and vertical connection systems.

For each of these, the protocol assumes the compilation of suitable modules, able to acquire information both of technical-constructive characteristics (masonry type, joints size, presence of abnormalities, transverse meshing, etc ..) and of decay (biological attacks, deformation, cracking, etc).

Interpolating matrix data arising from the decay evaluation (defect scoring), to constructive and typological quality data, for each subsystem, according to the following formu lation:

$\mathrm{ICCi}^{*}=\mathrm{f}\left(\mathrm{IDPi}^{*}, \mathrm{QTi}^{*}\right)(1)$

ICCi $^{*}=$ constructive conservation index $\mathrm{i}$-th

IDP = pathological degradation inde $\mathrm{x}$ i-th

$\mathrm{IQT}=$ technological quality inde $\mathrm{x}$ i-th

the ANVIV protocol is able to establish physical constructive conservation indexes, both local, for each subsystem:

$\mathrm{ICCv}^{*} \mathrm{~g}=\sum \mathrm{ICCi}^{*}(2)$

$\mathrm{ICCo}^{*} \mathrm{~g}=\sum \mathrm{ICCo}^{*}(3)$

$\mathrm{ICCo}^{*} \mathrm{~g}=\sum \mathrm{ICCo}^{*}(4)$

and global, for the entire masonry build ing:

$\mathrm{ICCg}=\mathrm{f}\left(\mathrm{ICCV} * \mathrm{~g} ; \mathrm{ICCO} * \mathrm{~g} ; \mathrm{ICCO}^{*} \mathrm{~g}\right)(5)$

and related class of conservative condition, within a rating scale, divided into five levels, from the highest performance class A, to class E (Fig. 1). This scale is fully defined following the standard made familiar by energy classification, with $20 \%$ intervals between a class and her next, in a range of values between 0 and 1 :

- Class A identifies conditions for which the individual local subsystem or build ing structure globally present a state of physical preservation as not to require detailed analytical assessment in the medium term. For buildings in this class are required interventions as ordinary periodic activities of inspection and control, or at most, whose chronological dilation does not involve a safety and preservation hazard for the structure;

- Class B states that the individual technological subsystem or the entire building organism manifests a conservation status that require analytical evaluation of detail in a fraction of the time compared to what is stipulated for class A. For buildings in this class are required interventions as ordinary periodic activities of inspection and control, or at most, whose chronological dilation does not involve a safety and preservation hazard for the structure in the mediu m/ short term;

- Class C identifies conditions for which the state of preservation requires analytical assessment of detail and consequent definition of recovery interventions. It is a class in which are required, in addition to ordinary periodic activities, recovery interventions delayable in the short term;

- Class D represents a preservation state which requires short-term analytical detail evaluation. In these cases are needed recovery interventions deriving from the analysis, to perform in a very short period, aimed at neutralizing situations of strong total or partial functional damage which, however, do not produce conditions of risk for the building organism physical conservation. In this context should also be inserted random interventions, whose priority realization is urgent and non-emergency;

- Class E identifies a status of significant criticality as to demand immediate-term analytical detail evaluation with high urgency. In these cases it is necessary to implement recovery interventions, enshrined in the analysis, to be carried out strictly with absolute urgency, aimed at neutralizing situations of strong total or partial functional loss which, however, do not produce hazardous conditions for the physical preservation of building structure.

All the parameters introduced in the protocol have been fully evaluated in correlation to their importance using methodologies such as Delphy online, and assigning relative weights using the Simos method. There were in fact involved a panel of 50 experts ( 25 engineers -25 architects) that deal with the recovery field, inviting them to make judgments on aspects such as the classification of sub-systems; degradation and technical-constructive parameters for typological solution. There were also performed sensitivity analysis on the dispersion and reliability of the data for each level of investigation performed in completing the questionnaires. 


\section{RESULTS}

ANVIV protocol validation, with specific reference to masonry structures with different geometric configuration in plan and elevation, has found experimental confirmation on the former monastery complex of Santa Chiara (Figs. 2-3).

This is a complex structure, developed at different moments in time between the sixteenth and the eighteenth century, disjointed into four macroblocks, properly identified on site (Fig. 4). The architectural morphological and constructive features, characterizing the monastery complex, make it more similar to an urban aggregate evolved over time, than to a classic historic building. In addition to the particular square shape, prolonged on one side, what characterizes the complex are the continuous aggregations to the original artefact, dating from 1573, so as to allow its conversion from a simple monastery complex to school building (northwest side), detention (east side) cinema theater (southwest). For each of the three macro-blocks in which remains disarticulated the whole monastery complex, in the face of an external regularity of the perimeter walls partitions, corresponds internally a subdivision as a result of the different transformations that have characterized the building over time. Finally the presence of mezzanine floors with different heights and positions, both on ground and first floor, causes elevation irregularity which can certainly not be neglected in the mechanical modeling of individual macro elements. The building structure has, in relation to its nominal life, a medium-low local conservation status as affected by local phenomena of obsolescence, as confirmed by pathological issues presence, which require appropriate and immediate consolidation work. In particular, for each of the three macroblocks, with the exception of the church block, not subject of experimentation, starting fro $m$ the compilation of the relative form modules, regarding both technicalconstructive characteristics and degradation for each of the three sub-systems, and interpolating data matrix deriving from the assessment of the state of degradation, defect scoring, to data on typologicalquality construction, it has come to define both a local building preservation index for vertical and horizontal load-bearing masonry solutions (Figs. 56) and a global index evaluation of the entire mac roblock.

But more precisely in accordance with the management models of conservative vulnerability, deriving from literature studies [4-5], from the experimentation emerges as the protocol is able to identify a macroblock interventions scale of priorities among: $\mathrm{B}$, in conservation clas $\mathrm{D}$, with a constructive retention index equal to 0.37692 , followed by the macroblock A, class $\mathrm{C}$, with index equal to 0.40492 , and finally the macroblock C, class $\mathrm{C}$, with an index of 0.4109 .

This ranking (Fig. 7) is perfectly compatible to comparable results obtained with static-seismic detail valuations, using a finite element model processing (Fig. 8). These verifications conducted on masonry solutions for buckling breaking mechanisms in the plane of the masonry or out-of-plane cutting, for cracking diagonal, have expressed their criticality to the $19.71 \%$ of masonry solutions for the block B, followed by $12.7 \%$ for A and finally a $4.5 \%$ for C. Although the evaluation conducted in this study, it is limited to a mere structural assessment, leaving out any pathological investigation of the individual subsystems degradation, have achieved appropriate results.

In particular, with a broader assessments, analyzing both pathological aspects of degradation and constructive aspects, the protocol is able to perceive fully critical locally and globally issues, like analytical evaluations of more properly sectoral detail.

\section{ConCLUS ION}

The research has led to the development of a tool that can be a valuable support for pre-analysis for the risk of loss mitigation of well-standardized structures in their plano-volumetric plant, with a deployment of limited resources and with a fair degree of approximation

This experimentation has certainly represented an important first step towards the applicability of ANVIV Protocol to traditional architectures, both monumental and ordinary, much more complex in their plant for their global system assessment. The ANVIV Protocol undoubtedly provides a further contribution in decision support practices for the risk of loss evaluation and reduction both seismic and more generally of historic buildings, essential both for urban and territorial vulnerability evaluation and in the management and scheduling of putting in safety interventions.

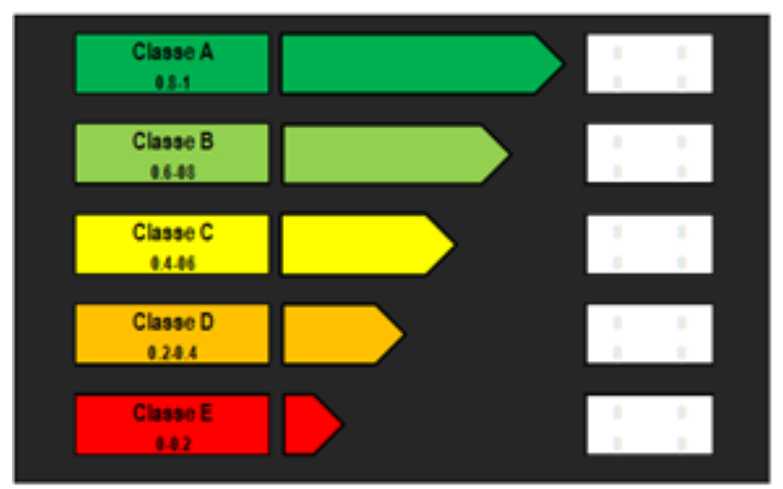

Figure 1: Constructive Conservation State Rating Scale. 


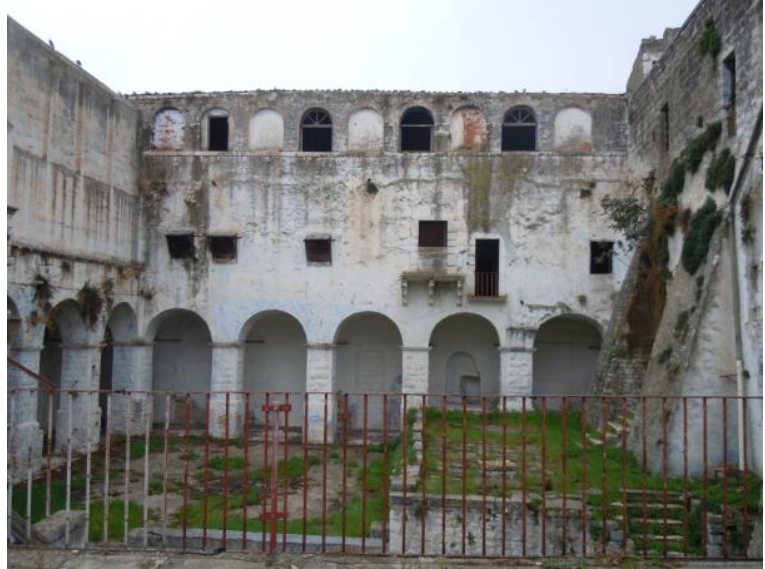

Figure 2: The monastery complex of Santa Chiara.

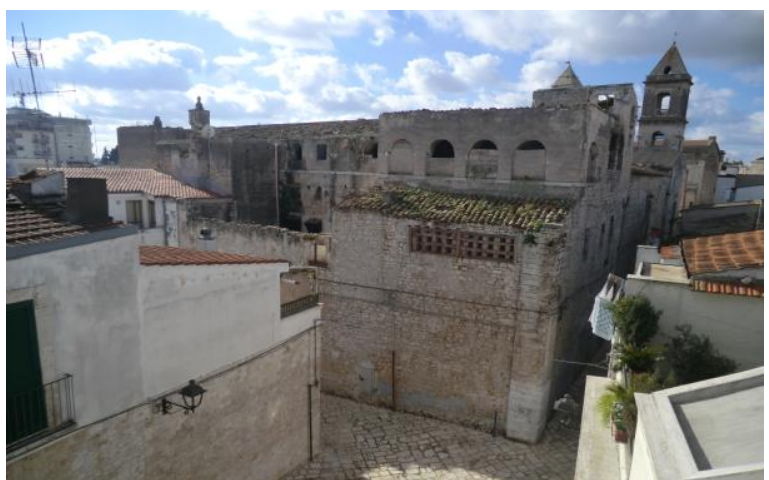

Figure 3: The monastery complex of Santa Chiara.

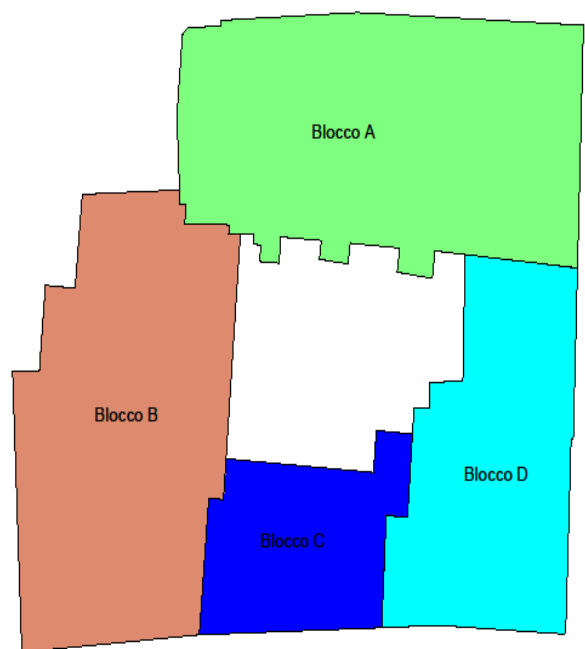

Figure 4: Monastery Complex Macroblock identification.
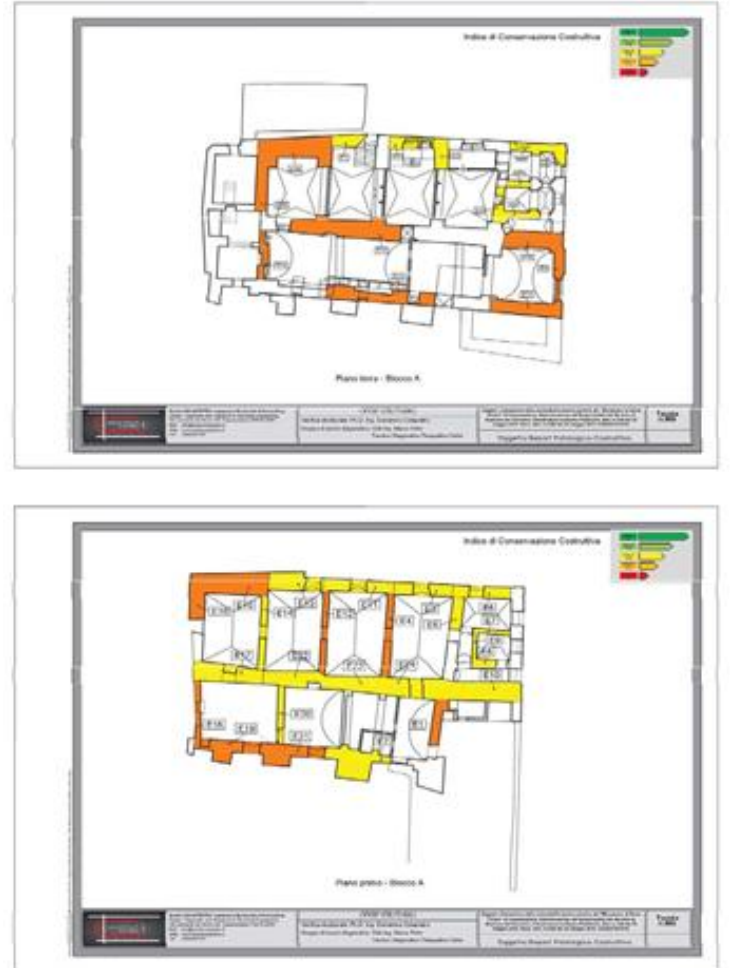

Figure 5: Constructive conservation Assessment of masonry vertical bearing solutions macroblock A.
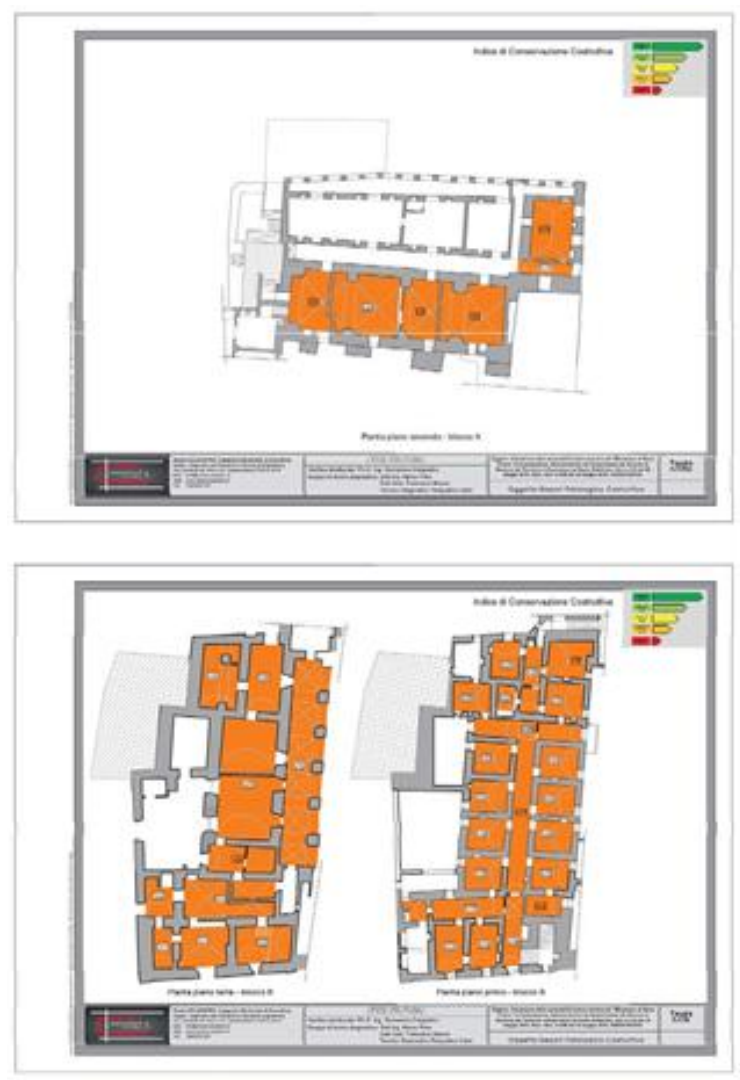

Figure 6: Constructive conservation Assessment of masonry horizontal bearing solutions macroblock B. 


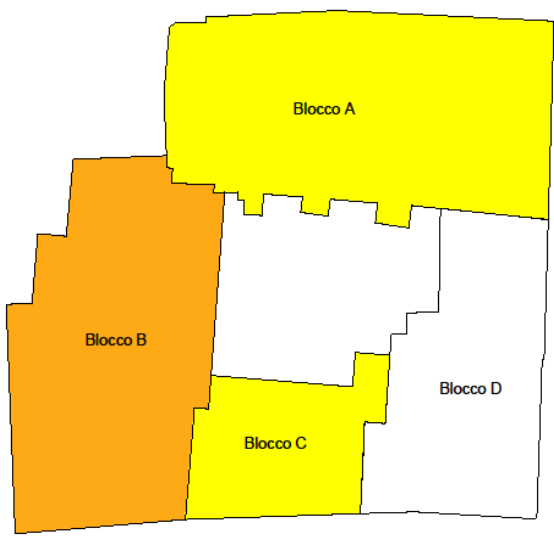

Figure 7: Global conservation status Evaluation of individual macroblocks.

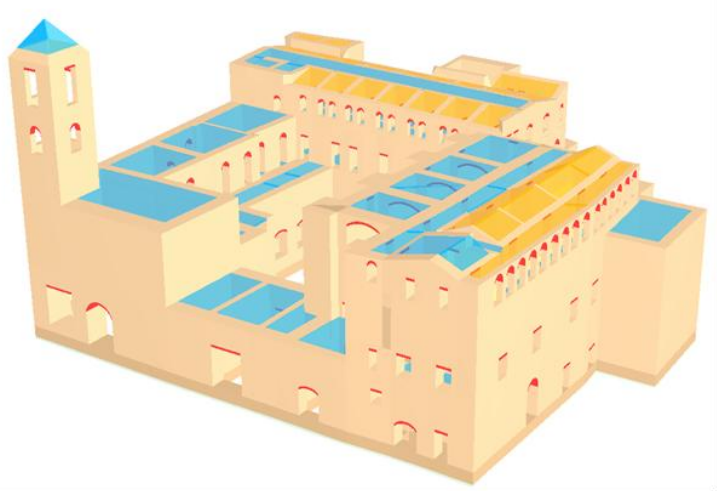

Figure 7: Macroele ments Mathematical Model of the monastery complex of Santa Chiara.

\section{REFERENCES}

[1] R. Cecchi, P. Gasparoli, Prevenzione e manutenzione per $i$ beni culturali edificati. Procedimenti scientifici per lo sviluppo delle attività ispettive: il caso studio delle aree archeologiche di Roma ed Ostia antica (Firen ze, Italy, A linea, 2010).

[2] M. Cilia, R. Cultrone, C. Occhipinti, Un metodo per la sicurezza dei centri storici. Interventi di miglioramento strutturale, Proc. Convegno Nazionale "Crolli ed affidabilità delle strutture civili”, Università degli studi di Messina, Messina, Italy, 20-22 April 2006.

[3] D. Colapietro, F. Fatiguso, Analysis of cooperative interpretation of constructive and degradation informations for the assessment of the state of physical preservation of traditional architectures in prevalent vertical development. The ANVIV protocol, Rivista TeMA: Tempo, Materia, Architettura, Vol. 1, N. 2, 2015.

[4] A. Borri, A. De Maria, S. Casaglia, Il metodo EAL-M per la classificazione sismica degli edifici in muratura esistenti: confronto tra diversi metodi proposti e prime considerazioni sull'estensione ad altre tipologie, Progettazione Sismica, n.2, Pavia, Italy, IUSS PRESS, 2014.

[5] G. M. Calvi, Un unico parametro per la valutazione delle prestazioni termiche e sismiche di un edificio, Progettazione Sismica n.2, Pavia, Italy, IUSS PRESS, 2013. 